\title{
SOME SPECIAL CASES OF BANG'S INEQUALITY
}

\author{
HENRY F. HUNTER
}

(Communicated by William J. Davis)

\begin{abstract}
Bang's inequality concerning the relative widths of $n$ planks covering a convex body is considered. Proofs are given that show just when equality occurs for two special cases in the plane, namely, two planks covering any convex body and three planks covering a triangle.
\end{abstract}

In 1932 Tarski [1] conjectured that the sum of the widths $w_{i}$ of $n$ planks (parallel strips) covering a convex body $K$ in $E^{2}$ is greater than or equal to the minimum width $W$ of $K$. This became a famous problem, which was finally proved by Bang [2] in 1950; subsequently Fenchel [3] simplified Bang's proof and extended it to $E^{d}$. Let us denote Tarski's inequality for $n$ planks (slabs) in $E^{d}$ by

$$
T(n, d): w_{1}+\cdots+w_{n} \geq W .
$$

At the end of his paper Bang posed the following as an "interesting problem": Is the sum of the relative widths $w_{i} / W_{i}$ greater than or equal to 1 ? $W_{i}$ is the width of $K$ in the same direction as the $i$ th plank. We denote Bang's inequality for $n$ planks in $E^{d}$ as

$$
B(n, d): w_{1} / W_{1}+\cdots+w_{n} / W_{n} \geq 1 .
$$

Ohmann [4] showed, by a continuity argument followed by an affine transformation, that the problem of proving $B(n, d)$ can be reduced to proving $B(n, n)$ for all $n$ with the planks mutually orthogonal and $w_{i}=1$.

We present proofs for two special cases in $E^{2}$ which are "complete" in the sense that they reveal just when equality occurs. In what follows, $r_{i}=w_{i}$, $R=r_{1}+\cdots+r_{n}$, and, as above, $i=1, \ldots, n$.

One approach to this problem is to give $K$ a special shape, e.g., a circle; or we could start with given planks and let $K$ vary, which is the approach taken in what follows.

Clearly only cases in which $K$ is maximal need be considered, and if $K$ is maximal, then it is necessarily a convex polygon each of whose sides lies on at least one corner point of one of the connected regions not covered by the given planks, since the Hahn-Banach-Tarski theorem assures that through each such point there is a line bounding $K$ and those lines form a convex polygon containing $K$ and covered by the planks.

Received by the editors July 1, 1991.

1991 Mathematics Subject Classification. Primary 52A10, 52A40.

(C) 1993 American Mathematical Society $0002-9939 / 93 \$ 1.00+\$ .25$ per page 


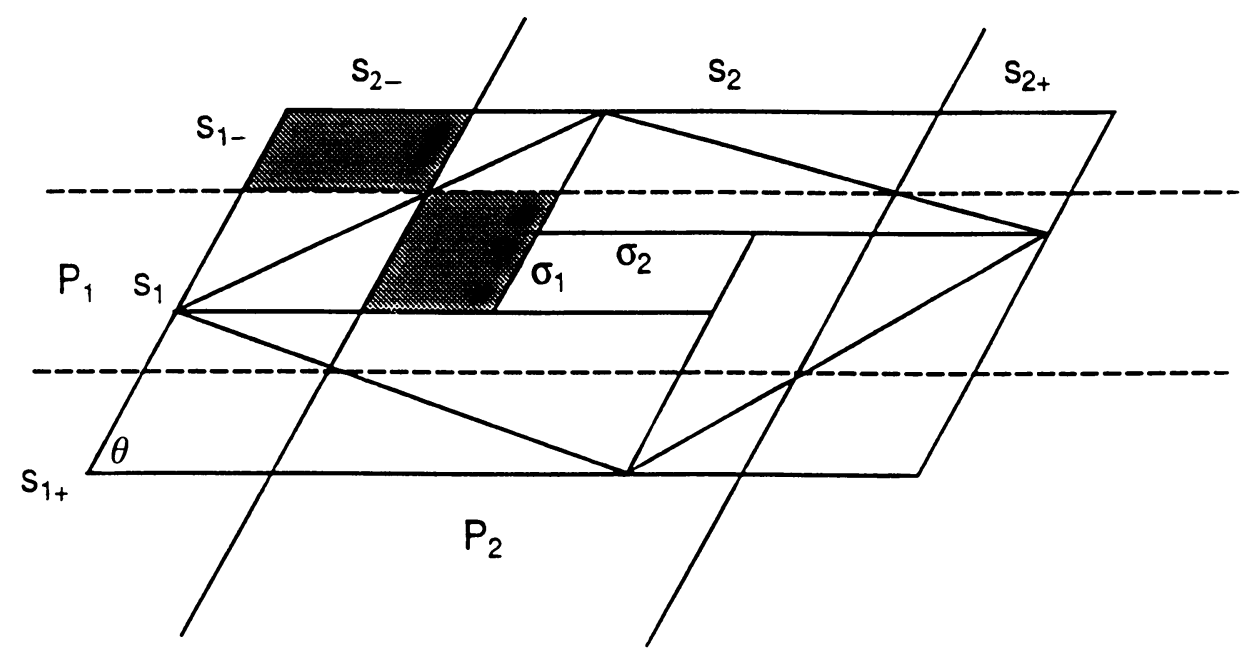

FIGURE 1

For the case $n=2$, in Figure 1

$$
S_{i}=s_{i-}+s_{i}+s_{i+}, \quad r_{i}=s_{i} / S_{i}, \quad \rho_{i}=\sigma_{i} / S_{i} .
$$

The shaded areas are equal. Considering the parallelogram containing them, and similarly the parallelograms at each of the other three corners, gives (after dividing by $\sin \theta$ )

$$
\left(S_{1}-s_{1}\right)\left(S_{2}-s_{2}\right)=s_{1-} s_{2-}+s_{1-} s_{2+}+s_{1+} s_{2-}+s_{1+} s_{2+}=s_{1} s_{2}-\sigma_{1} \sigma_{2} .
$$

Dividing by $S_{1} S_{2}$ yields

$$
\left(1-r_{1}\right)\left(1-r_{2}\right)=r_{1} r_{2}-\rho_{1} \rho_{2},
$$

which simplifies to

$$
R=1+\rho_{1} \rho_{2} .
$$

Thus $R=1$ when and only when one diagonal of $K$ is parallel to the plank that covers it, in which case the other diagonal is also parallel to its plank (by Desargue's theorem). Since $\rho_{1}$ and $\rho_{2}$ change sign simultaneously, their product remains nonnegative. Hence $R \geq 1$.

There is as yet no general solution for $n=3$; but a special case, that of three planks covering a triangle, can be solved as follows. First make an affine transformation to take the triangle into an equilateral triangle whose sides are of unit length. Label the lengths of the segments of those sides as in Figure 2. Regard $t_{1}, t_{2}, t_{3}$ as independent variables and find $s_{1}$ in terms of $t$ :

$$
(A+B) s_{1}=B+t_{2}\left(1-t_{2}\right)\left(1-t_{1}-t_{2}\right)
$$

where $A=\left(1-t_{1}\right)\left(1-t_{2}\right)\left(1-t_{3}\right)$ and $B=\left(1-t_{1}-t_{3}\right)\left(1-t_{2}-t_{1}\right)\left(1-t_{3}-t_{2}\right)$. Let $T=t_{1}+t_{2}+t_{3}$ and $S=s_{1}+s_{2}+s_{3}$. Then $s_{2}$ and $s_{3}$ are found by cyclic permutation. Summing (after quite a bit of juggling) yields

$$
R-1=(1-T)^{2} /(2-T) \geq 0 \text { since } T \leq 3 / 2 .
$$

Thus $R=1$ when and only when $T=1$. 


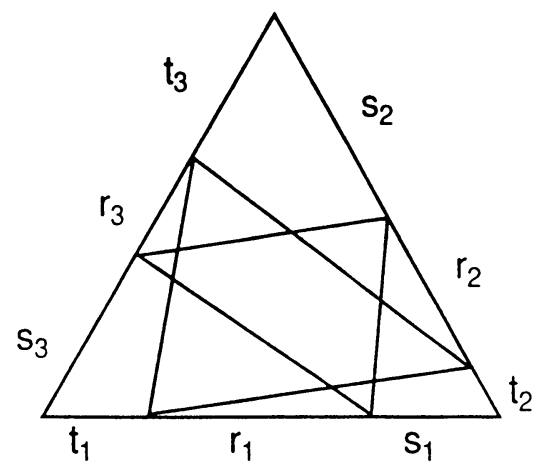

FIGURE 2

Other relations are $(2-T)(2-S)=1$ and $R-1=(1-T)(S-1)$, wherein the factors $(1-T)$ and $(S-1)$ change sign simultaneously.

\section{ACKNOWLEDGMENT}

I wish to thank Professor S. Ross of Rensselaer Polytechnic Institute for his assistance in preparing this paper.

\section{REFERENCES}

1. A. Tarski, Further remarks about the degree of equivalence of polygons, Odbitka z Parametru 2 (1932), 310-314.

2. T. Bang, A solution of the "plank problem", Proc. Amer. Math. Soc. 2 (1951), 990-993. MR 13:769

3. W. Fenchel, On Th. Bang's solution of the plank problem, Mat. Tidsskr. B 1951 (1951), 49-51. MR 13: 863.

4. D. Ohmann, Eine abschätzung für die dicke bei Überdeckung durch konvexe Körper, J. Reine Angew. Math. 190 (1952), 125-128. MR 14:788

400 Central Avenue, Apartment 13A, Albany, New York 12206-2243 